\title{
Spinal anesthesia: how can we improve patient satisfaction?
}

\author{
Jong Seok Lee \\ Department of Anesthesiology and Pain Medicine, Yonsei University College of Medicine, Seoul, Korea
}

Since spinal anesthesia was introduced in clinics about 100 years ago, many studies have examined whether spinal anesthesia decreases operating time, mortality, cardiovascular morbidity, number of patients requiring a blood transfusion, deep vein thrombosis and pulmonary embolism, pain, opioidrelated adverse effects, and the length of hospital stay when compared with general anesthesia. Macfarlane et al. [1] performed a systemic review of the contemporary literature comparing general anesthesia and/or systemic analgesia with regional anesthesia for total knee arthroplasty. They did not suggest a difference in blood loss or duration of surgery. However, regional anesthesia reduced postoperative pain and opioid-related adverse effects in patients undergoing total knee arthroplasty. Length of hospital stay may also be reduced and rehabilitation facilitated by regional anesthesia compared with general anesthesia. They found insufficient evidence from randomized, controlled trials alone to conclude whether anesthetic technique influenced mortality, cardiovascular morbidity other than postoperative hypotension, or the incidence of deep vein thrombosis and pulmonary embolism in the setting of routine thromboprophylaxis.

In this issue of the journal, Rhee et al. [2] prospectively evaluated factors affecting dissatisfaction and refusal of spinal anesthesia. Anesthesiologists have a duty to inform patients about risks and alternatives to anesthetic methods. It is the basic spirit of medical ethics to allow patients to choose an anesthesia method. Anesthesiologists frequently hold a biased view when they choose the anesthetic method rather than obtain the opinion of patients, and they have a variety of reasons for choosing a given anesthetic technique. A technique may be chosen simply because we know it; it may be a favorite technique or a less familiar technique chosen to maintain competence/experience or to teach. Other reasons are a request from patients or surgeons [3]. If anesthesiologist allow patients to decide the anesthetic method, in many cases the patients say "I don't know. It's up to you”; "Is spinal anesthesia painful?", "I don't want to awaken during the operation", “Will I feel pain after the operation?", or "Does spinal anesthesia cause back pain?". Therefore, the anesthesiologists must explain fully about the applicable anesthesia method and help patients to decide the procedure by their own volition. If a patient can not decide their anesthetic method, anesthesiologists must choose it considering the patient's situation.

In a study by Rhee et al. [2] 44/1,191 patients (3.7\%) revealed dissatisfaction with spinal anesthesia. The reasons for the dissatisfaction were backache (29.5\%), postoperative nausea and vomiting (PONV; 20.4\%), pain at the puncture site (15.9\%), inadequate analgesia (13.6\%), consciousness during the operation (6.8\%), postdural puncture headache (4.5\%), transient neurologic symptoms (4.5\%), and urinary retention (4.5\%). In that study, postoperative backache was the most common cause of dissatisfaction. However, back pain may not be directly related to spinal anesthesia and is almost always associated with pre-existing back pain $[4,5]$. Backache after spinal anesthesia is almost always associated with positions during the operation, surgical trauma, operation time, age, pregnancy, needle type, the number of punctures, a different bed, or long bed rest other than preadmission to the hospital $[4,6]$. Therefore, if the anesthesiologist had explained to the patient that back pain was not directly related to the spinal anesthesia, in most cases, the results of their study would change. PONV after spinal anesthesia is often due to fentanyl administered

Corresponding author: Jong Seok Lee, M.D., Department of Anesthesiology and Pain Medicine, Yonsei University College of Medicine, Gangnam Severance Hospital, 612, Eonjuro, Gangnam-gu, Seoul 135-720, Korea. Tel: 82-2-2019-3524, Fax: 82-2-3463-0940, E-mail: jonglee@yuhs.ac (c) This is an open-access article distributed under the terms of the Creative Commons Attribution Non-Commercial License (http:// creativecommons.org/licenses/by-nc/3.0/), which permits unrestricted non-commercial use, distribution, and reproduction in any medium, provided the original work is properly cited. 
with local anesthetics. It is difficult to say that PONV is a side effect of spinal anesthesia. It can increase the satisfaction rate not to mix opioids with local anesthetics when it is known that PONV will cause a problem to patients. Rhee et al. [2] reported that the dissatisfaction rate from spinal anesthesia was $3.7 \%$. Other studies have demonstrated higher rates such as $3.8-17 \%$ $[7,8]$. A patient's satisfaction is associated with multiple factors including the methods and attitudes of the investigators, social circumstances, and the relationship between the patient and doctor. I think that the patient-doctor relationship is the most important factor when physicians care for their patients, and good rapport can affect patient satisfaction.

Paresthesia and pain at the puncture site are dependent on the skillfulness of the physician, the number of punctures, and age and gender of the patient. Sedation during an operation with spinal anesthesia is frequently required and is an important factor to increase satisfaction rate. Midazolam, propofol, and dexmedetomidine are used for this purpose. The main uses for sedation in regional anesthesia are to reduce anxiety and improve patient comfort and cooperation [9].

In conclusion, some factors increase the satisfaction rate of patients who are undergoing an operation with spinal anesthesia. First, a patient's opinion is very important when deciding the anesthetic method. Secondly, the anesthesiologists must fully explain about spinal anesthesia before surgery. Thirdly, the anesthesiologists should have good rapport with their patients. Finally, the anesthesiologist should manipulate the patient skillfully and sedate the patients if they want.

\section{References}

1. Macfarlane AJ, Prasad GA, Chan VW, Brull R. Does regional anesthesia improve outcome after total knee arthroplsty? Clin Orthop Relat Res 2009; 467: 2379-402.

2. Rhee WJ, Chung CJ, Lim YH, Lee KH, Lee SC. Factors in patient dissatisfaction and refusal regarding spinal anesthesia. Korean J Anesthesiol 2010; 59: 260-4.

3. Buckley N. Regional vs general anaesthesia in orthopaedics. Can J Anaesth 1993; 40: R104-12.

4. Horlocker TT, McGregor DG, Matsushige DK, Schroeder DR, Besse JA. A retrospective review of 4767 consecutive spinal anesthetics: central nervous system complications. Anesth Analg 1997; 84: 57884.

5. Schwabe K, Hopf HB. Persistent back pain after spinal anesthesia in the non-obstetric setting: incidence and predisposing factors. Br J Anaesth 2001; 86: 535-9.

6. Choi JG, In JY, Shin HI. Analysis of factors related to patient refusal of spinal anesthesia. Korean J Anesthesiol 2009; 56: 156-61.

7. Charuluxananan S, Sriprajittichai P, Sirichotvithyakorn P, Rodanant O, Kyokong O. Factors related to patient satisfaction regarding spinal anesthesia. J Med Assoc Thai 2003; 86 Suppl 2: S338-43.

8. Siddiqi R, Jafri SA. Maternal satisfaction after spinal anaesthesia for cesarean deliveries. J Coll Physicians Surg Pak 2009; 19: 77-80.

9. Hu P, Harmon D, Frizelle H. Patient comfort during regional anesthesia. J Clin Anesth 2007; 19: 67-74. 